\title{
(c) (1) \\ Obesity: An independent protective factor for localized renal cell carcinoma in a systemic inflammation state
}

\author{
Zhenhua Liu ${ }^{1}$, Haifeng Wang ${ }^{2}$, Yuke Chen ${ }^{1}$, Jie Jin ${ }^{1}$, Wei Yu ${ }^{1}$ \\ ${ }^{1}$ Department of Urology, Peking University First Hospital and Institute of Urology, Peking University, \\ National Urological Cancer Center, Beijing, China; ${ }^{2}$ Department of Anesthesiology, Peking University \\ First Hospital, Peking University, Beijing, China
}

\section{ABSTRACT}

Objectives: To explore the prognostic value of obesity (measured by BMI) on RCC in a systemic inflammation state.

Patients and Methods: Clinicopathological and hematological data of 540 surgically treated Chinese localized RCC patients between 2005 and 2010 were retrospectively collected. Found by receiver operating characteristic (ROC) curve for cancer-specific survival (CSS), the optimal cutoff values of neutrophil-lymphocyte ratio (NLR, an indicator of systemic inflammation state) and BMI were 2.12 and 23.32, respectively. Survival curves were drawn using Kaplan-Meier method. Univariate and multivariate Cox regression analyses were used to evaluate the prognostic value of BMI in localized RCC patients with different NLR.

Results: Overall, 36 patients died with a median follow-up of 70 months. Median overall survival (OS) was 66 months and the 5-year OS rate was 92.7\%. In the multivariate analysis of total patients, higher BMI was an independent protective factor for CSS in total patients $(p=0.048)$. While in systemic inflammation subgroup (high NLR subgroup) patients, higher BMI (obesity) turned out to be an independent protective factor for both CSS $(p=0.025)$ and RFS ( $p=0.048)$.

Conclusion: In localized RCC patients, obesity was an independent protective factor for CSS and RFS in a systemic inflammation state.

\section{ARTICLE INFO}

Zhenhua Liu

http://orcid.org/0000-0002-3152-1007

\section{Keywords:}

Obesity; Carcinoma;

Inflammation

Int Braz J Urol. 2020; 46: 585-98

Submitted for publication:

April 04, 2019

Accepted after revision:

August 11, 2019

Published as Ahead of Print:

October 30, 2019

\section{INTRODUCTION}

Renal cell carcinoma (RCC) is the most common malignancy of kidney, accounting for $2 \%-3 \%$ of all adult malignancies (1). $20 \%-40 \%$ of localized RCC patients still suffered from cancer recurrence or metastasis even after surgery treatment, despite the significant improvement of RCC therapy (2). Thus, it is of importance to find effective prognostic factors to facilitate progress in treatment strategy.
Obesity is a widely accepted risk factor for the onset of RCC $(3,4)$. As an indicator of obesity, body mass index (BMI) was widely studied for its effect on the prognosis of RCC. Nevertheless, although obesity increases the incidence of RCC, several previous studies have shown that RCC patients with higher BMI at diagnosis might have better survival outcomes than those with normal or lower BMI levels (5-7). However, some investigators fail to confirm the existence of such association $(8,9)$. Although increasing evidence supports that higher 
BMI is a favorable prognostic factor of RCC, this topic has not been thoroughly explored.

Increased neutrophil-lymphocyte ratio (NLR) is significantly associated with insulin resistance (IR), which is considered the common cause of impaired glucose tolerance, diabetes, dyslipidemia, hypertensive diseases and obesity (10). And accumulating evidence suggests that high NLR might be an adverse prognostic factor in metastatic RCC patients treated with interferon, interleukin-2 or sunitinib (11-13). However, studies regarding the prognostic value of NLR in non-metastatic RCC remain sparse.

NLR is an easily accessible index and high NLR has been proposed as an indicator of systemic inflammatory response, which is independently associated with clinical outcomes of various cancers (14). A systemic inflammatory state may be established long before metastases become clinically evident (15). Thus, it is of importance to study the prognostic effect of BMI under systemic inflammation state.

\section{MATERIALS AND METHODS}

\section{Study population}

Our retrospective study included 540 patients with localized renal cell carcinoma who underwent curative surgeries in Peking University First Hospital between 2005 and 2010. Patient's collection was based on the following inclusion criteria: (1) patients who were pathologically diagnosed with localized RCC (pT1-2NOM0, p: pathological grading) after surgery, (2) patients with complete information about BMI and NLR, (3) patients who had at least one effective follow-up. Patients were excluded if they had any of the following condition: (1) patients with previously diagnosed cancers or autoimmune diseases, (2) patients with incomplete clinical or pathological data, (3) patients who underwent previous chemotherapy and/or radiation therapy. This study was approved by the institutional ethics committee of Peking University First Hospital. As a retrospective analysis of routine data, a waiver of written informed consent was granted from the ethics committee. Patient records/information was anonymized and de-identified prior to analysis.
Clinical and pathological data collection

Clinicopathological and hematological data including gender (female or male), age (years old), height $(\mathrm{m})$, weight $(\mathrm{kg})$, cancer related symptoms (absent or present), histological subtype (clear cell or non-clear cell), Fuhrman nuclear grade (1-2 or 3-4), tumor necrosis (no or yes), tumor laterality (left or right), tumor size $(\leq 7 \mathrm{~cm}$ or $>7 \mathrm{~cm}$, equals to T1 or T2 in TNM staging system), surgical procedures (partial or radical), neutrophil counts and lymphocyte counts were collected from medical records in the Department of Urology, Peking University First Hospital. Pathological TNM stage for each RCC was determined according to the AJCC 2002 TNM staging system. Patients were closely followed up after discharge with regular post-operative tests. BMI $\left(\mathrm{kg} / \mathrm{m}^{2}\right)$ was calculated based on the measurements of height and weight at diagnosis. NLR was calculated as preoperative neutrophil counts divided by lymphocyte counts. The optimal cut-off value of BMI (23.32) and NLR (2.12) were determined according to the receiver operating characteristic (ROC) curves (shown in supplementary Figures S.1 and S.2 of cancer-specific survival (CSS). According to the Asian and Chinese standard of obesity, the normal ceiling of BMI is $23-24(16,17)$, and our cut-off value (23.32) falls in this range.

\section{Indicators of prognosis}

Overall survival (OS), cancer-specific survival (CSS) and recurrence-free survival (RFS) were used as indicators of prognosis of the localized RCC patients in the study. OS, CSS, RFS were the intervals between the date of surgery treatment and (1) the date of death or last follow-up, (2) cancer-related death or last follow-up, (3) radiologic or histological confirmation of cancer recurrence or last follow-up.

\section{Statistical analysis}

The clinicopathological characteristics between groups with different BMI were compared using chi-square test. Kaplan-Meier survival curves were compared by using the log-rank test. BMI and other variables with $\mathrm{P}<0.1$ in univariate analysis were included in the multivaria- 
te Cox proportional hazards regression model, and $\mathrm{P}<0.05$ (labeled with '*') was regarded as statistically significant. Also, by using Cox proportional hazards regression models, we obtained the hazard ratios (HRs) and 95\% confidence intervals (CIs) from the survival time. Data were analyzed using IBM SPSS statistics software (version 22.0, SPSS Inc., Chicago, IL, USA) for Microsoft Windows. Pictures were drawn by GraphPad Prism (version7, Graphpad software, Inc., La Jolla, CA, USA, Figure 1) and IBM SPSS statistics software (Figure-2).

Figure 1 - Correlation analysis between BMI and NLR.

\section{BMI NLR correlations}

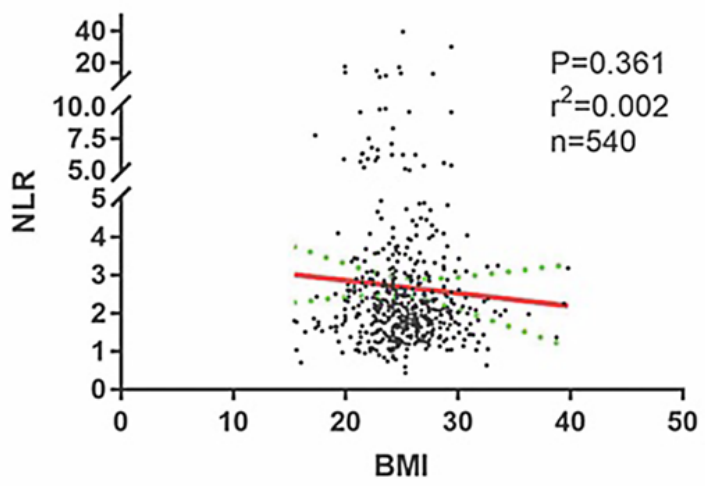

No correlation could be found between BMI and NLR $(p>0.05)$.

Abbreviation: $\mathbf{B M I}=$ body mass index, $\mathbf{N L R}=$ neutrophil-lymphocyte ratio

\section{RESULTS}

\section{Cohort characteristics}

In total, 400 men and 140 women with localized RCC were included in the study with a mean age of $54 \pm 13.4$ years old. $48.3 \%$ (261/540) of the tumors were located on the left side. Only $53(9.8 \%)$ patients manifested cancer related symptoms (backache, hematuria and/or abdominal mass). Most patients $(88.9 \%, 480 / 540)$ suffered from clear cell carcinoma. Patients whose tumor size was bigger than $7 \mathrm{~cm}$ accounted for less than 10\%. Using 23.32 as the cutoff value of BMI, 145 (26.9\%) and 395 (73.1\%) patients were respectively stratified into the reference group (low BMI group BMI <23.32) and high BMI group (BMI $\geq 23.32$ ). Differences in gender were found between reference group and high BMI group $\mathrm{p}=0.006)$. No differences were found between reference group and high BMI group in terms of age, tumor laterality, cancer related symptoms presence, histology, tumor size, Fuhrman nuclear grade, tumor necrosis or NLR. 283 and 257 patients were respectively categorized into low and high NLR group, according to the cutoff value of NLR at 2.12 (Table-1). Correlation analysis found no correlation between NLR and BMI (Figure-1).

\section{Survival analysis on BMI and NLR}

We used OS, CSS and RFS as the indicators of prognosis to estimate the association of BMI

Figure 2 - Survival curves stratified by BMI at the level of 23.32 in total patients (Kaplan-Meier method).
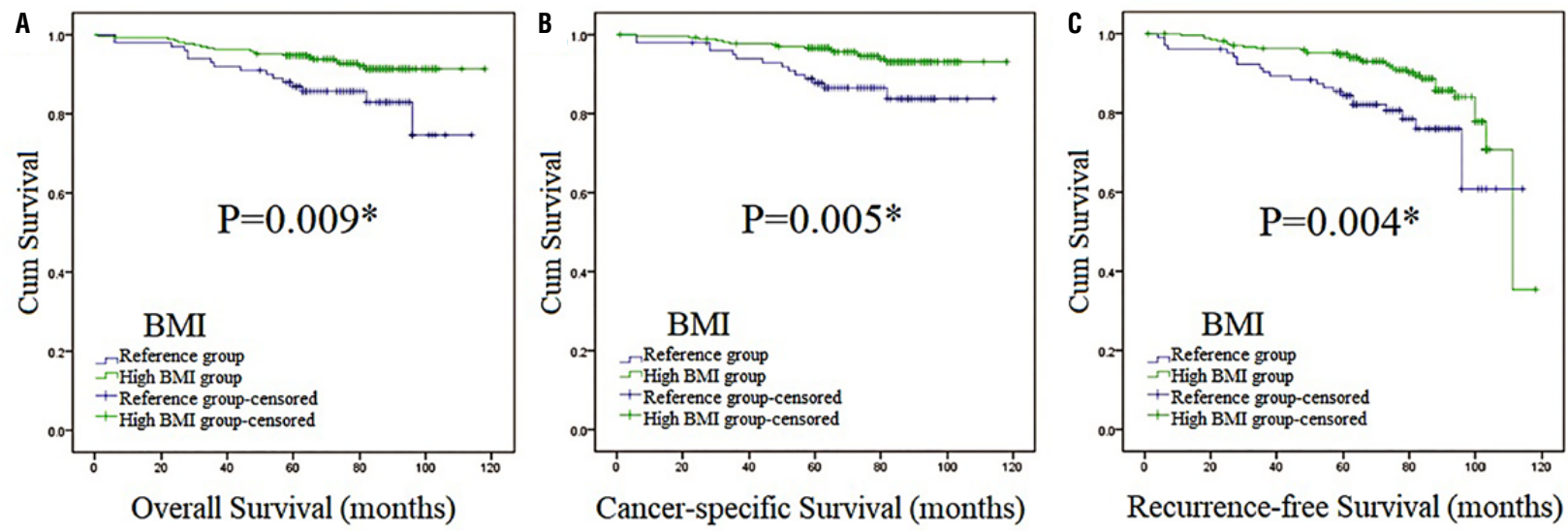

Survival curves of OS (Figure 2A), CSS (Figure 2B) and RFS (Figure 2C) in total patients.

*: $p<0.05$; Abbreviation: BMI=body mass index 
Table 1 - Distribution of clinicopathological factors stratified by preoperative BMI.

\begin{tabular}{|c|c|c|c|c|}
\hline \multirow{2}{*}{ Characteristics } & All patients & $\mathrm{BMI}<23.32$ & $\mathrm{BMI} \geq 23.32$ & \multirow{2}{*}{$P$ value } \\
\hline & $\mathrm{N}=540(\mathrm{~N} \%)$ & $\mathrm{N}=145$ (N\%) & $\mathrm{N}=395(\mathrm{~N} \%)$ & \\
\hline Age & & & & 0.184 \\
\hline$<60$ & $352(65.2)$ & $88(60.7)$ & $264(66.8)$ & \\
\hline$\geq 60$ & $188(34.8)$ & $57(39.3)$ & $131(33.2)$ & \\
\hline Gender & & & & $0.006^{*}$ \\
\hline Male & $400(74.1)$ & $95(65.5)$ & $305(77.2)$ & \\
\hline Female & $140(25.9)$ & $50(34.5)$ & $90(22.8)$ & \\
\hline Tumor laterality & & & & 0.428 \\
\hline Left & $261(48.3)$ & $66(45.5)$ & $195(49.4)$ & \\
\hline Right & $279(51.7)$ & $79(54.5)$ & $200(50.6)$ & \\
\hline Cancer related symptoms & & & & 0.219 \\
\hline Absent & $487(90.2)$ & $127(87.6)$ & $360(91.1)$ & \\
\hline Present & $53(9.8)$ & $18(12.4)$ & $35(8.9)$ & \\
\hline \multicolumn{5}{|l|}{ Surgical procedures } \\
\hline Partial resection & $157(29.1)$ & $40(27.6)$ & $118(29.9)$ & 0.605 \\
\hline Radical resection & $383(70.9)$ & $105(72.4)$ & $277(70.1)$ & \\
\hline Histology & & & & 0.131 \\
\hline Clear cell & $480(88.9)$ & $124(85.5)$ & $356(90.1)$ & \\
\hline Non-clear cell & $60(11.1)$ & $21(14.5)$ & $39(9.9)$ & \\
\hline Tumor size & & & & 0.066 \\
\hline$\leq 7$ (T1NOMO) & $496(91.9)$ & $128(88.3)$ & $368(93.2)$ & \\
\hline$>7$ (T2NOMO) & $44(8.1)$ & $17(11.7)$ & $27(6.8)$ & \\
\hline Fuhrman Grade & & & & 0.085 \\
\hline $1-2$ & 479 (88.7) & $123(84.8)$ & $356(90.1)$ & \\
\hline $3-4$ & $61(11.3)$ & $22(15.2)$ & $39(9.9)$ & \\
\hline Tumor necrosis & & & & 0.163 \\
\hline Absent & $462(85.6)$ & $119(82.1)$ & $343(86.8)$ & \\
\hline Present & $78(14.4)$ & $26(17.9)$ & $52(13.2)$ & \\
\hline NLR & & & & 0.244 \\
\hline$\leq 2.12$ & $283(52.4)$ & $70(48.3)$ & $213(53.9)$ & \\
\hline$>2.12$ & 257 (47.6) & $75(51.7)$ & $182(46.1)$ & \\
\hline
\end{tabular}

*: $p<0.05$; Abbreviations: $\mathbf{B M I}=$ body mass index; $\mathbf{N L R}=$ neutrophil-lymphocyte ratio.

The data of histology, tumor size, Fuhrman Grade and tumor necrosis were obtained from pathological findings of surgical specimens. 
and the clinical outcomes of patients with localized RCC. The median follow-up was 70 months (in the range of 1-118). Overall, 36 patients died, with 16 and 20 in the reference group and high BMI group, respectively. Median OS was 66 months (in the range of 1-118) and the 5-year OS rate was 92.7\%. Kaplan-Meier (K-M) curves indicated that differences were found between reference group and high BMI group in OS, CSS and RFS, establishing that BMI was associated with OS, CSS and RFS in our study (Figure-2). Also, high BMI group had higher survival curves of OS, CSS and RFS than the reference group, which indicated that high BMI group might have better OS,
CSS and RFS. On the other hand, patients with high NLR had worse OS and CSS than low NLR according to survival curves (Figure-3), which indicated they had worse OS and CSS. Then, subgroup analysis was performed by stratifying subjects by NLR at the level of 2.12. As we could see in Figure-S.3, BMI was associated with RFS ( $p=0.010)$. While in high NLR group patients (Figure 4), association was found between BMI and CSS ( $p=0.021)$. In Figure-5, K-M curves were drawn using a combination of BMI and NLR, of which high BMI-low NLR subgroup had best survival outcomes, while low BMI-high NLR subgroup was the worst.

Figure 3 - Survival curves stratified by NLR at the level of 2.12 in total patients (Kaplan-Meier method).
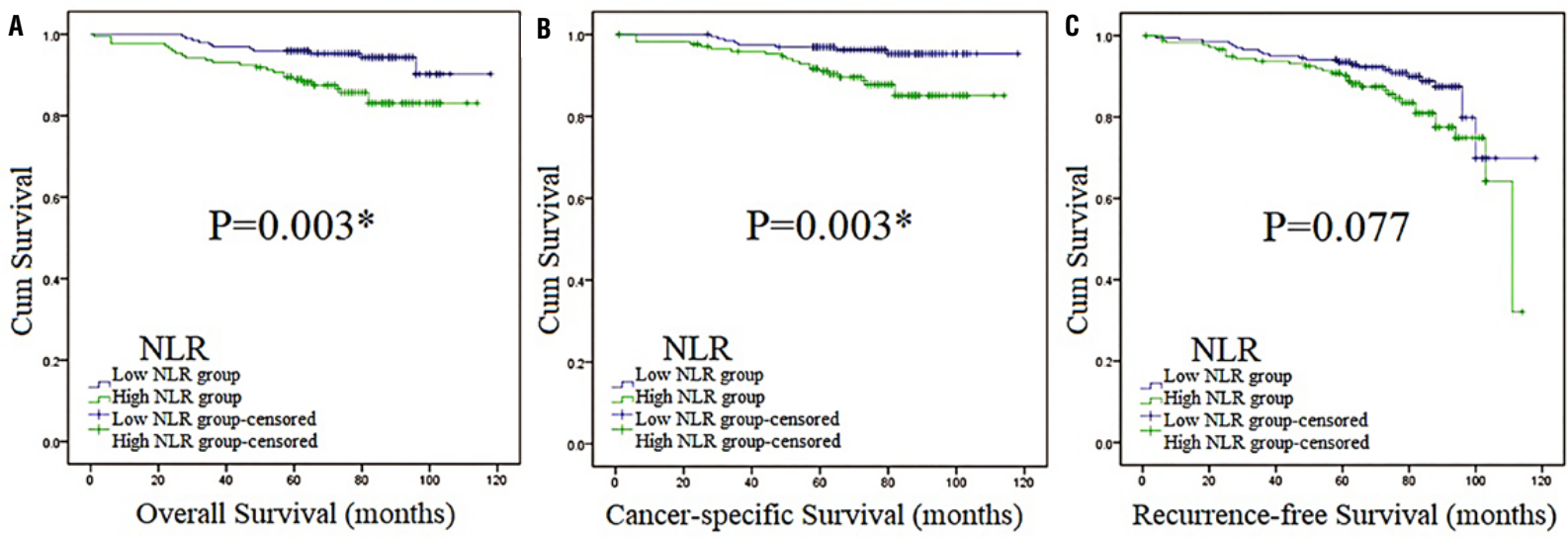

Survival curves of OS (Figure 3A), CSS (Figure 3B) and RFS (Figure 3C) in total patients.

Abbreviation: $\mathbf{N L R}=$ neutrophil-lymphocyte ratio.

*:p $<0.05$

Figure 4 - Survival curves stratified by BMI at the level of 23.32 in high NLR patients (Kaplan-Meier method).
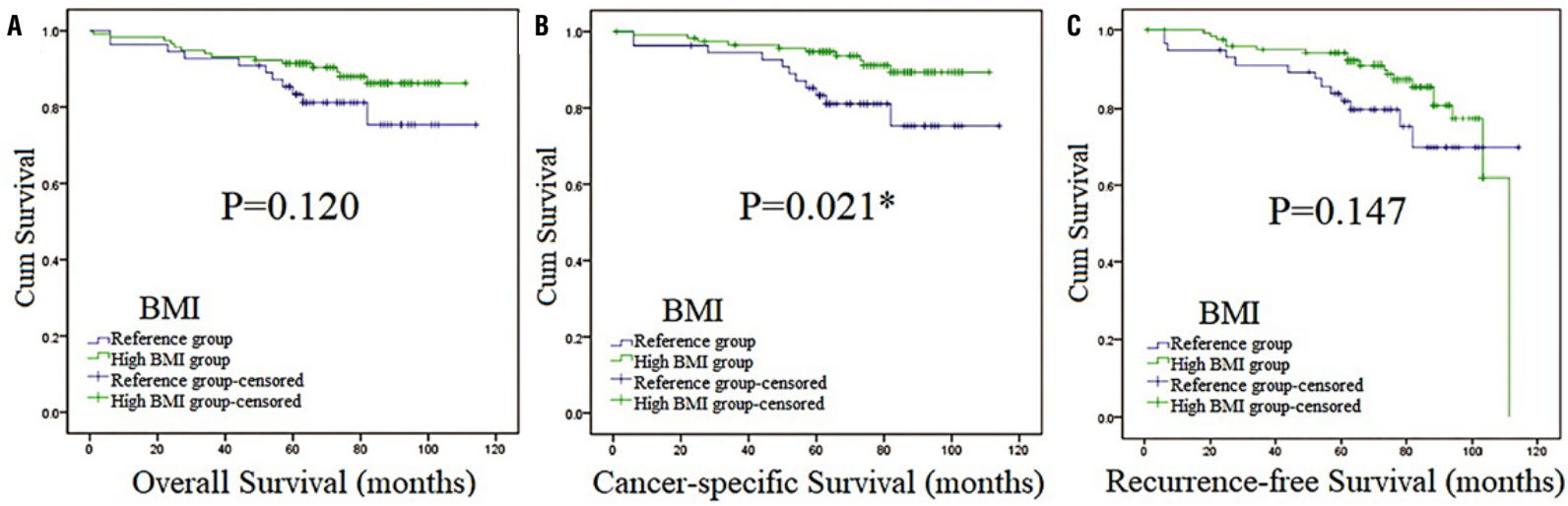

Survival curves of OS (Figure 4A), CSS (Figure 4B) and RFS (Figure 4C) in high NLR patients. Abbreviations: $\mathbf{B M I}=$ body mass index; $\mathbf{N L R}=$ neutrophil-lymphocyte ratio.

*:p $<0.05$ 
Figure 5 - Survival curves stratified by BMI-NLR at the level of 23.32 (BMI) and 2.12 (NLR) in total patients (KaplanMeier method).
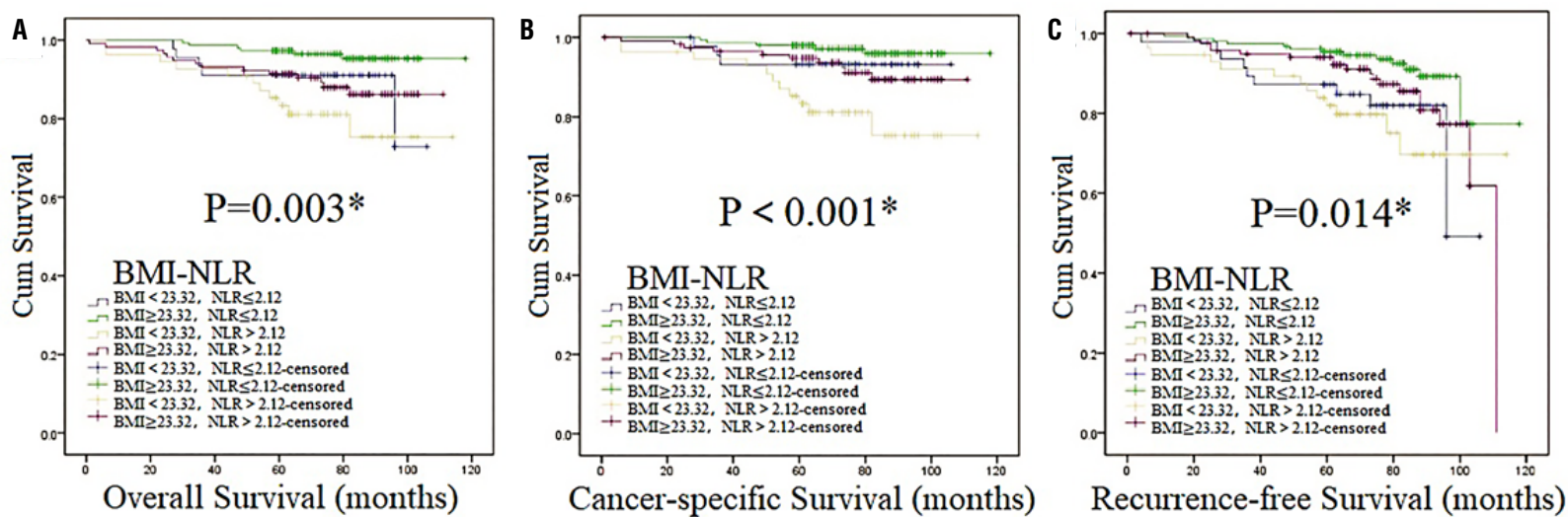

Survival curves of OS (Figure 5A), CSS (Figure 5B) and RFS (Figure 5C) in total patients

Abbreviations: $\mathbf{B M I}=$ body mass index; $\mathbf{N L R}=$ neutrophil-lymphocyte ratio.

${ }^{*}: p<0.05$

Univariate and multivariate analysis

Univariate Cox regression analyses of factors for OS, CSS and RFS were shown in Tables 2-4. In univariate analysis of total patients, larger tumor size ( $>7$ vs. $\leq 7 \mathrm{~cm})$ and lower BMI $(<23.32$ vs. $\geq 23.32$ ) were associated with poorer OS, CSS and RFS (all $p<0.05$ ). Older age ( $\geq 60$ vs. $<60$ years) and higher NLR ( $>2.12$ vs. $\leq 2.12)$ were correlated with lower OS ( $p<0.001, p=0.005$, respectively) and CSS ( $p=0.003, p=0.005$, respectively). Presence of cancer related symptoms was associated with worse CSS $(p=0.012)$ and RFS $(p=0.001)$. Radical nephrectomy (radical vs. partial) and higher Fuhrman grade (3-4 vs. 1-2) were correlated with poorer OS ( $\mathrm{p}=0.047, \mathrm{p}=0.004$, respectively) and RFS ( $p<0.030, p<0.001$, respectively). Gender, tumor laterality, histology and tumor necrosis were not associated with OS, CSS or RFS (all p >0.05).

Subgroup univariate analysis revealed that in low NLR group patients, older age remained its association with lower OS $(p=0.004)$ and CSS $(p=0.024)$. Higher Fuhrman nuclear grade had correlation with poorer OS $(p=0.030)$ and RFS $(p=0.001)$. Larger BMI value was associated with better RFS $(p=0.014)$. While in high NLR group (systemic inflammation state) patients, older age was associated with worse OS ( $\mathrm{p}=0.027)$. Manifestation of cancer related symptoms and larger tu- mor size were correlated with worse CSS $(\mathrm{p}=0.027$, $\mathrm{p}=0.038$, respectively) and RFS (both $\mathrm{p}<0.001$ ), but not OS. Radical nephrectomy (radical vs. partial) was associated with poorer RFS ( $p=0.043)$. Larger BMI value was correlated with better CSS $(\mathrm{p}=0.027)$.

Outcomes of multivariate Cox regression analysis of OS, CSS and RFS are listed in Tables $2-4$. In the multivariate analysis of total patients, manifestation of cancer related symptoms and larger tumor size were independent risk factors for OS ( $p=0.015 ; p=0.045$, respectively), CSS ( $p=0.003$; $\mathrm{p}=0.023$, respectively) and RFS $(\mathrm{p}=0.006 ; \mathrm{p}<0.001$, respectively). Older age and higher NLR had independent adverse effects on OS ( $p<0.001 ; p=0.006$, respectively) and CSS ( $\mathrm{p}=0.003 ; \mathrm{p}=0.005$, respectively). While higher BMI was only an independent protective factor for CSS $(\mathrm{HR}=0.474,95 \% \mathrm{CI}$ : 0.226-0.994, $\mathrm{p}=0.048$ ).

Furthermore, subgroup multivariate analysis turned out that in low NLR subgroup, older age was an independent risk factor for OS $(p=0.005)$ and CSS $(p=0.024)$, higher Fuhrman grade was an independent adverse predictor for OS ( $p=0.0499)$ and RFS ( $p=0.001)$. While in high NLR group (systemic inflammation state) patients, presence of cancer related symptoms and larger tumor size became independent risk factors for 
Table 2 - Univariate and multivariate analysis of OS in total patients, low NLR and high NLR subgroups.

\begin{tabular}{|c|c|c|c|c|c|c|c|c|c|c|c|c|}
\hline \multirow{3}{*}{ Characteristics } & \multicolumn{4}{|c|}{ Total } & \multicolumn{4}{|c|}{ Low NLR } & \multicolumn{4}{|c|}{ High NLR (systemic inflammation state) } \\
\hline & \multicolumn{2}{|c|}{ Univariate analysis } & \multicolumn{2}{|c|}{ Multivariate analysis } & \multicolumn{2}{|c|}{ Univariate analysis } & \multicolumn{2}{|c|}{ Multivariate analysis } & \multicolumn{2}{|c|}{ Univariate analysis } & \multicolumn{2}{|c|}{ Multivariate analysis } \\
\hline & $\mathrm{HR}(95 \% \mathrm{Cl})$ & $P$ & $\mathrm{HR}(95 \% \mathrm{Cl})$ & $P$ & $\mathrm{HR}(95 \% \mathrm{Cl})$ & $\mathrm{P}$ & $\mathrm{HR}(95 \% \mathrm{Cl})$ & $P$ & $\mathrm{HR}(95 \% \mathrm{Cl})$ & $P$ & $\mathrm{HR}(95 \% \mathrm{Cl})$ & $P$ \\
\hline $\begin{array}{l}\text { Gender (female } \\
\text { vs. male) }\end{array}$ & $\begin{array}{l}0.574 \\
(0.252- \\
1.311)\end{array}$ & 0.188 & & & $\begin{array}{c}0.790(0.210- \\
2.978)\end{array}$ & 0.728 & & & $\begin{array}{c}0.517(0.177- \\
1.507)\end{array}$ & 0.227 & & \\
\hline $\begin{array}{l}\text { Age (<60 vs. } \\
\geq 60)\end{array}$ & $\begin{array}{l}3.734 \\
(1.867- \\
7.468)\end{array}$ & $<0.001^{*}$ & $\begin{array}{c}4.010(1.987- \\
8.091)\end{array}$ & $<0.001$ & $\begin{array}{c}9.482(2.048- \\
43.911)\end{array}$ & $0.004^{*}$ & $\begin{array}{r}9.040 \\
(1.948- \\
41.949)\end{array}$ & $0.005^{\star}$ & $\begin{array}{c}2.471(1.110- \\
5.501)\end{array}$ & $0.027^{\star}$ & $\begin{array}{c}3.348 \\
(1.424- \\
7.873)\end{array}$ & $0.006^{*}$ \\
\hline $\begin{array}{l}\text { Tumor } \\
\text { laterality (left } \\
\text { vs. right) }\end{array}$ & $\begin{array}{l}1.417 \\
(0.730- \\
2.749)\end{array}$ & 0.303 & & & $\begin{array}{c}1.330 \text { (0.406- } \\
4.361)\end{array}$ & 0.638 & & & $\begin{array}{c}1.379(0.619- \\
3.072)\end{array}$ & 0.431 & & \\
\hline $\begin{array}{l}\text { Cancer related } \\
\text { symptoms } \\
\text { (absent vs. } \\
\text { present) }\end{array}$ & $\begin{array}{r}2.322 \\
(0.965- \\
5.589)\end{array}$ & 0.06 & $\begin{array}{c}3.073(1.241- \\
7.614)\end{array}$ & $0.015^{\star}$ & $\begin{array}{c}2.116(0.455- \\
9.828)\end{array}$ & 0.339 & & & $\begin{array}{c}2.736(0.938- \\
7.978)\end{array}$ & 0.065 & $\begin{array}{c}3.872 \\
(1.263- \\
11.869)\end{array}$ & $0.018^{*}$ \\
\hline $\begin{array}{l}\text { Surgical } \\
\text { procedures } \\
\text { (partial vs. } \\
\text { radical) }\end{array}$ & $\begin{array}{c}2.603 \\
(1.012- \\
6.699)\end{array}$ & $0.047^{\star}$ & & & $\begin{array}{c}5.677(0.726- \\
44.349)\end{array}$ & 0.098 & & & $\begin{array}{c}1.465(0.503- \\
4.271)\end{array}$ & 0.484 & & \\
\hline $\begin{array}{l}\text { Tumor size ( } \leq 7 \\
\text { vs. }>7)\end{array}$ & $\begin{array}{c}2.778 \\
(1.156- \\
6.677)\end{array}$ & $0.022^{\star}$ & $\begin{array}{c}2.483(1.022- \\
6.030)\end{array}$ & $0.045^{\star}$ & $\begin{array}{c}2.358(0.302- \\
18.444)\end{array}$ & 0.414 & & & $\begin{array}{c}2.290(0.859- \\
6.108)\end{array}$ & 0.098 & $\begin{array}{l}2.856 \\
(1.047- \\
7.793)\end{array}$ & $0.040^{*}$ \\
\hline $\begin{array}{l}\text { Histology } \\
\text { (clear cell vs. } \\
\text { non-clear cell) }\end{array}$ & $\begin{array}{l}1.085 \\
(0.384- \\
3.070)\end{array}$ & 0.877 & & & $\begin{array}{c}0.675(0.146- \\
3.131)\end{array}$ & 0.616 & & & $\begin{array}{c}1.424(0.336- \\
6.040)\end{array}$ & 0.632 & & \\
\hline $\begin{array}{l}\text { Tumor necrosis } \\
\text { (no vs yes) }\end{array}$ & $\begin{array}{c}1.563 \\
(0.684- \\
3.573)\end{array}$ & 0.29 & & & $\begin{array}{c}3.346(0.879- \\
12.731)\end{array}$ & 0.077 & & & $\begin{array}{c}0.935(0.321- \\
2.726)\end{array}$ & 0.902 & & \\
\hline $\begin{array}{l}\text { Fuhrman grade } \\
\text { (1-2 vs. } 3-4)\end{array}$ & $\begin{array}{l}3.051 \\
(1.433- \\
6.498)\end{array}$ & $0.004^{\star}$ & & & $\begin{array}{c}4.377(1.153- \\
16.615)\end{array}$ & $0.030^{*}$ & $\begin{array}{l}3.887 \\
(1.001- \\
15.098)\end{array}$ & $0.0499^{*}$ & $\begin{array}{c}2.200(0.877- \\
5.520)\end{array}$ & 0.093 & & \\
\hline $\begin{array}{l}\text { NLR ( } \leq 2.12 \text { vs. } \\
>2.12)\end{array}$ & $\begin{array}{c}2.762 \\
(1.359- \\
5.614)\end{array}$ & $0.005^{\star}$ & $\begin{array}{c}2.761(1.340- \\
5.690)\end{array}$ & $0.006^{\star}$ & & & & & & & & \\
\hline $\begin{array}{l}\text { BMI (<23.32 } \\
\text { vs. } \geq 23.32)\end{array}$ & $\begin{array}{c}0.428 \\
(0.222- \\
0.828)\end{array}$ & $0.012^{\star}$ & & & $\begin{array}{c}0.334(0.102- \\
1.097)\end{array}$ & 0.071 & & & $\begin{array}{c}0.540(0.244- \\
1.191)\end{array}$ & 0.127 & & \\
\hline
\end{tabular}

${ }^{*}: p<0.05$; Abbreviations: $\mathbf{B M I}=$ body mass index; $\mathbf{N L R}=$ neutrophil-lymphocyte ratio; $\mathbf{H R}=$ hazard ratio; $\mathbf{C l = c o n f i d e n c e ~ i n t e r v a l ; ~} \mathbf{0 S}=0$ verall survival; /=not significant; Blank space $=$ not done. 
Table 3 - Univariate and multivariate analysis of CSS in total patients, low NLR and high NLR subgroups.

\begin{tabular}{|c|c|c|c|c|c|c|c|c|c|c|c|c|}
\hline \multirow{3}{*}{ Characteristics } & \multicolumn{4}{|c|}{ Total } & \multicolumn{4}{|c|}{ Low NLR } & \multicolumn{4}{|c|}{ High NLR (systemic inflammation state) } \\
\hline & \multicolumn{2}{|c|}{ Univariate analysis } & \multicolumn{2}{|c|}{ Multivariate analysis } & \multicolumn{2}{|c|}{ Univariate analysis } & \multicolumn{2}{|c|}{ Multivariate analysis } & \multicolumn{2}{|c|}{ Univariate analysis } & \multicolumn{2}{|c|}{ Multivariate analysis } \\
\hline & $\mathrm{HR}(95 \% \mathrm{Cl})$ & $P$ & $\begin{array}{c}\text { HR } \\
(95 \% \mathrm{Cl})\end{array}$ & $P$ & $\mathrm{HR}(95 \% \mathrm{Cl})$ & $P$ & $\mathrm{HR}(95 \% \mathrm{Cl})$ & $P$ & HR $(95 \% \mathrm{Cl})$ & $P$ & $\mathrm{HR}(95 \% \mathrm{Cl})$ & $P$ \\
\hline $\begin{array}{l}\text { Gender (female vs. } \\
\text { male) }\end{array}$ & $\begin{array}{c}0.752 \\
(0.321- \\
1.761)\end{array}$ & 0.511 & & & $\begin{array}{c}0.711 \\
(0.143- \\
3.522)\end{array}$ & 0.676 & & & $\begin{array}{c}0.839(0.307- \\
2.292)\end{array}$ & 0.732 & & \\
\hline Age $(<60$ vs. $\geq 60)$ & $\begin{array}{c}3.084 \\
(1.456- \\
6.530)\end{array}$ & $0.003^{\star}$ & $\begin{array}{c}3.024 \\
(1.339- \\
6.827)\end{array}$ & $0.003^{\star}$ & $\begin{array}{c}6.326 \\
(1.276- \\
31.365)\end{array}$ & $0.024^{*}$ & $\begin{array}{c}6.326 \\
(1.276- \\
31.365)\end{array}$ & $0.024^{*}$ & $\begin{array}{c}2.198(0.926- \\
5.218)\end{array}$ & 0.074 & $\begin{array}{c}2.991 \\
(1.165- \\
7.682)\end{array}$ & $0.023^{*}$ \\
\hline $\begin{array}{l}\text { Tumor laterality } \\
\text { (left vs. right) }\end{array}$ & $\begin{array}{l}1.251 \\
(0.602- \\
2.601)\end{array}$ & 0.549 & & & $\begin{array}{l}1.103 \\
(0.276- \\
4.414)\end{array}$ & 0.89 & & & $\begin{array}{c}1.236(0.521- \\
2.936)\end{array}$ & 0.631 & & \\
\hline $\begin{array}{l}\text { Cancer related } \\
\text { symptoms (absent } \\
\text { vs. present) }\end{array}$ & $\begin{array}{l}3.146 \\
(1.280- \\
6.847)\end{array}$ & $0.012^{\star}$ & $\begin{array}{c}4.597 \\
(1.746- \\
12.101)\end{array}$ & $0.003^{*}$ & $\begin{array}{c}3.328 \\
(0.669- \\
16.547)\end{array}$ & 0.142 & & & $\begin{array}{c}3.415(1.148- \\
10.164)\end{array}$ & $0.027^{\star}$ & $\begin{array}{c}5.285 \\
(1.624- \\
17.200)\end{array}$ & $0.006^{*}$ \\
\hline $\begin{array}{l}\text { Surgical } \\
\text { procedures (partial } \\
\text { vs. radical) }\end{array}$ & $\begin{array}{l}2.599 \\
(0.904- \\
7.469)\end{array}$ & 0.076 & & & $\begin{array}{l}3.851 \\
(0.474- \\
31.308)\end{array}$ & 0.207 & & & $\begin{array}{c}1.689(0.497- \\
5.736)\end{array}$ & 0.401 & & \\
\hline $\begin{array}{l}\text { Tumor size ( } \leq 7 \\
\text { vs. }>7)\end{array}$ & $\begin{array}{c}3.641 \\
(1.481- \\
8.949)\end{array}$ & $0.005^{\star}$ & $\begin{array}{c}2.889 \\
(1.160- \\
7.195)\end{array}$ & $0.023^{*}$ & $\begin{array}{l}3.363 \\
(0.413- \\
27.387)\end{array}$ & 0.257 & & & $\begin{array}{c}2.901(1.061- \\
7.930)\end{array}$ & $0.038^{*}$ & $\begin{array}{c}3.968 \\
(1.420- \\
11.086)\end{array}$ & $0.009^{\star}$ \\
\hline $\begin{array}{l}\text { Histology (clear } \\
\text { cell vs. non-clear } \\
\text { cell) }\end{array}$ & $\begin{array}{c}1.168 \\
(0.353- \\
3.859)\end{array}$ & 0.799 & & & $\begin{array}{c}0.435 \\
(0.088- \\
2.156)\end{array}$ & 0.308 & & & $\begin{array}{c}2.484(0.333- \\
18.514)\end{array}$ & 0.375 & & \\
\hline $\begin{array}{l}\text { Tumor necrosis (no } \\
\text { vs yes) }\end{array}$ & $\begin{array}{l}1.032 \\
(0.359- \\
2.969)\end{array}$ & 0.953 & & & $\begin{array}{c}2.802 \\
(0.563- \\
13.933)\end{array}$ & 0.208 & & & $\begin{array}{c}0.521(0.121- \\
2.237)\end{array}$ & 0.38 & & \\
\hline $\begin{array}{l}\text { Fuhrman grade } \\
\text { (1-2 vs. } 3-4)\end{array}$ & $\begin{array}{c}2.410 \\
(0.980- \\
5.928)\end{array}$ & 0.055 & & & $\begin{array}{l}4.076 \\
(0.821- \\
20.242)\end{array}$ & 0.086 & & & $\begin{array}{c}1.622(0.544- \\
4.831)\end{array}$ & 0.385 & & \\
\hline $\begin{array}{l}\operatorname{NLR}(\leq 2.12 \text { vs. } \\
>2.12)\end{array}$ & $\begin{array}{c}3.212 \\
(1.422- \\
7.254)\end{array}$ & $0.005^{\star}$ & $\begin{array}{c}3.360 \\
(1.453- \\
7.769)\end{array}$ & $0.005^{\star}$ & & & & & & & & \\
\hline $\begin{array}{l}\text { BMI (<23.32 } \\
\text { vs. } \geq 23.32)\end{array}$ & $\begin{array}{l}0.363 \\
(0.175- \\
0.753)\end{array}$ & $0.006^{\star}$ & $\begin{array}{l}0.474 \\
(0.226- \\
0.994)\end{array}$ & $0.048^{\star}$ & $\begin{array}{l}0.461 \\
(0.110- \\
1.933)\end{array}$ & 0.29 & & & $\begin{array}{c}0.378(0.160- \\
0.893)\end{array}$ & $0.027^{\star}$ & $\begin{array}{l}0.367 \\
(0.153- \\
0.879)\end{array}$ & $0.025^{*}$ \\
\hline
\end{tabular}

*:p<0.05; Abbreviations: $\mathbf{B M I = b o d y ~ m a s s ~ i n d e x ; ~} \mathbf{N L R}=$ neutrophil-lymphocyte ratio; $\mathbf{H R}=$ hazard ratio; $\mathbf{C l}=$ confidence interval; $\mathbf{C S S}=$ cancer specific survival; /=not significant; Blank space=not done. 
Table 4 - Univariate and multivariate analysis of RFS in total patients, low NLR and high NLR subgroups.

\begin{tabular}{|c|c|c|c|c|c|c|c|c|c|c|c|c|}
\hline \multirow{3}{*}{ Characteristics } & \multicolumn{4}{|c|}{ Total } & \multicolumn{4}{|c|}{ Low NLR } & \multicolumn{4}{|c|}{ High NLR (systemic inflammation state) } \\
\hline & \multicolumn{2}{|c|}{ Univariate analysis } & \multicolumn{2}{|c|}{ Multivariate analysis } & \multicolumn{2}{|c|}{ Univariate analysis } & \multicolumn{2}{|c|}{$\begin{array}{l}\text { Multivariate } \\
\text { analysis }\end{array}$} & \multicolumn{2}{|c|}{ Univariate analysis } & \multicolumn{2}{|c|}{$\begin{array}{l}\text { Multivariate } \\
\text { analysis }\end{array}$} \\
\hline & $\begin{array}{c}\mathrm{HR} \\
(95 \% \mathrm{Cl})\end{array}$ & $P$ & $\begin{array}{c}\mathrm{HR} \\
(95 \% \mathrm{Cl})\end{array}$ & $P$ & $\mathrm{HR}(95 \% \mathrm{Cl})$ & $P$ & $\begin{array}{c}\text { HR } \\
(95 \% \mathrm{Cl})\end{array}$ & $P$ & $\mathrm{HR}(95 \% \mathrm{Cl})$ & $P$ & $\begin{array}{c}\mathrm{HR} \\
(95 \% \mathrm{Cl})\end{array}$ & $P$ \\
\hline $\begin{array}{l}\text { Gender } \\
\text { (female vs. } \\
\text { male) }\end{array}$ & $\begin{array}{c}0.707 \\
(0.380- \\
1.315)\end{array}$ & 0.273 & & & $\begin{array}{c}0.836(0.346- \\
2.022)\end{array}$ & 0.692 & & & $\begin{array}{c}0.613(0.251- \\
1.469)\end{array}$ & 0.282 & & \\
\hline $\begin{array}{l}\text { Age (<60 vs. } \\
\geq 60)\end{array}$ & $\begin{array}{c}1.494 \\
(0.876- \\
2.548)\end{array}$ & 0.14 & & & $\begin{array}{c}2.277(1.019- \\
5.088)\end{array}$ & 0.045 & & & $\begin{array}{c}1.027(0.498- \\
2.117)\end{array}$ & 0.943 & & \\
\hline $\begin{array}{l}\text { Tumor } \\
\text { laterality (left } \\
\text { vs. right) }\end{array}$ & $\begin{array}{c}0.922 \\
(0.545- \\
1.558)\end{array}$ & 0.761 & & & $\begin{array}{c}1.065(0.478- \\
2.371)\end{array}$ & 0.878 & & & $\begin{array}{c}0.840(0.419- \\
1.685)\end{array}$ & 0.624 & & \\
\hline $\begin{array}{l}\text { Cancer related } \\
\text { symptoms } \\
\text { (absent vs. } \\
\text { present) }\end{array}$ & $\begin{array}{c}3.065 \\
(1.610- \\
5.833)\end{array}$ & $0.001^{\star}$ & $\begin{array}{c}2.501 \\
(1.294- \\
4.836)\end{array}$ & $0.006^{*}$ & $\begin{array}{c}2.061(0.701- \\
6.055)\end{array}$ & 0.189 & & & $\begin{array}{c}4.429(1.968- \\
9.967)\end{array}$ & $<0.001 * t$ & $\begin{array}{c}5.671 \\
(2.393- \\
13.440)\end{array}$ & $<0.001^{*}$ \\
\hline $\begin{array}{l}\text { Surgical } \\
\text { procedures } \\
\text { (partial vs. } \\
\text { radical) }\end{array}$ & $\begin{array}{c}2.210 \\
(1.080- \\
4.522)\end{array}$ & $0.030^{\star}$ & & & $\begin{array}{c}1.370(0.567- \\
3.312)\end{array}$ & 0.484 & & & $\begin{array}{c}4.398(1.046- \\
18.500)\end{array}$ & $0.043^{*}$ & $\begin{array}{c}4.151 \\
(0.929- \\
18.545)\end{array}$ & 0.062 \\
\hline $\begin{array}{l}\text { Tumor size ( } \leq 7 \\
\text { vs. }>7 \text { ) }\end{array}$ & $\begin{array}{l}4.823 \\
(2.656- \\
8.757)\end{array}$ & $<0.001^{*}$ & $\begin{array}{c}3.837 \\
(2.048- \\
7.187)\end{array}$ & $<0.001^{*}$ & $\begin{array}{c}3.049(0.900- \\
10.332)\end{array}$ & 0.073 & & & $\begin{array}{c}5.737(2.757- \\
11.937)\end{array}$ & $<0.001^{\star}$ & $\begin{array}{l}4.574 \\
(2.125- \\
9.848)\end{array}$ & $<0.001^{*}$ \\
\hline $\begin{array}{l}\text { Histology } \\
\text { (clear cell vs. } \\
\text { non-clear cell) }\end{array}$ & $\begin{array}{l}1.769 \\
(0.639- \\
4.897)\end{array}$ & 0.273 & & & $\begin{array}{c}1.134(0.336- \\
3.825)\end{array}$ & 0.84 & & & $\begin{array}{c}3.706(0.505- \\
27.184)\end{array}$ & 0.198 & & \\
\hline $\begin{array}{l}\text { Tumor } \\
\text { necrosis (no } \\
\text { vs yes) }\end{array}$ & $\begin{array}{l}1.476 \\
(0.741- \\
2.941)\end{array}$ & 0.268 & & & $\begin{array}{c}2.602(0.955- \\
7.094)\end{array}$ & 0.062 & & & $\begin{array}{c}0.911(0.346- \\
2.402)\end{array}$ & 0.851 & & \\
\hline $\begin{array}{l}\text { Fuhrman } \\
\text { grade (1-2 vs. } \\
3-4)\end{array}$ & $\begin{array}{c}2.957 \\
(1.626- \\
5.378)\end{array}$ & $<0.001^{*}$ & $\begin{array}{c}1.990 \\
(1.051- \\
3.768)\end{array}$ & $0.035^{\star}$ & $\begin{array}{c}4.923(1.996- \\
12.144)\end{array}$ & $0.001^{\star}$ & $\begin{array}{c}4.923 \\
(1.996- \\
12.144)\end{array}$ & $0.001^{*}$ & $\begin{array}{c}1.885(0.833- \\
4.266)\end{array}$ & 0.128 & & \\
\hline $\begin{array}{l}\text { NLR }(\leq 2.12 \\
\text { vs. }>2.12)\end{array}$ & $\begin{array}{c}1.604 \\
(0.944- \\
2.726)\end{array}$ & 0.081 & & & & & & & & & & \\
\hline $\begin{array}{l}\text { BMI (<23.32 } \\
\text { vs. } \geq 23.32)\end{array}$ & $\begin{array}{c}0.467 \\
(0.274- \\
0.797)\end{array}$ & $0.005^{\star}$ & & & $\begin{array}{c}0.358(0.157- \\
0.815)\end{array}$ & $0.014^{*}$ & & & $\begin{array}{c}0.595(0.292- \\
1.211)\end{array}$ & 0.152 & $\begin{array}{c}0.477 \\
(0.229- \\
0.994)\end{array}$ & $0.048^{\star}$ \\
\hline
\end{tabular}

*: $p<0.05$; Abbreviations: $\mathbf{B M I}=$ body mass index; $\mathbf{N L R}=$ neutrophil-lymphocyte ratio; $\mathbf{H R}=$ hazard ratio; $\mathbf{C l}=$ confidence interval; $\mathbf{R F S}=$ recurrence-free survival; /=not significant; Blank space=not done. 
OS, CSS and RFS (cancer related symptoms presence: $\mathrm{p}=0.018, \mathrm{p}=0.006, \mathrm{p}<0.001$, respectively; tumor size: $p=0.040, p=0.009, p<0.001$, respectively). Also, older age was an independent adverse predictor for OS $(\mathrm{p}=0.006)$ and CSS $(\mathrm{p}=0.023)$. Interestingly, higher BMI turned out to be an independent protective factor for CSS (HR $=0.367$, 95\%CI: 0.153-0.879, $\mathrm{p}=0.025)$ and RFS (HR=0.477, 95\%CI: 0.229-0.994, $\mathrm{p}=0.048$ ) in high NLR group (systemic inflammation state) patients.

\section{DISCUSSION}

This study evaluated the prognostic value of BMI both in total patients and in systemic inflammation state patients. To the best of our knowledge, this is the first study to explore the prognostic value of obesity for localized renal cell carcinoma in a systemic inflammation state.

\section{BMI and RCC prognosis}

Obesity has emerged as a significant adverse predictor for RCC in previous studies. People with an increased BMI have two to three folds increased risk for developing RCC (18). The hypothetical explanations for the increased risk included the alteration of the insulin-like growth factor system, lipid peroxidation, high levels of estrogen, hypertension and the malfunction of immune system. However, there seems to be a paradox: obesity increases the risk of RCC but in the meantime, it is associated with improved tumor prognosis. In our study, obesity was an independent favorable prognostic factor for CSS in total patients. The results were in line with some Asian studies. Jeon et al. found overweight and obese Korean patients with RCC had more favorable prognosis than those with a normal BMI (19). Similar researches by Awakura et al. also reported that a BMI of $23 \mathrm{~kg} /$ $\mathrm{m}^{2}$ or more favorably affected the prognosis of Japanese RCC subjects, although BMI did not differ significantly with respect to stage or grade (20).

Similar results have also been obtained in some studies about western RCC patients. Yu et al. suggested that prognosis was no worse and may even be better among obese patients with RCC (21). In a study of 400 patients with non-metastatic, node-negative RCC conducted by Kamat et al., overweight and obese patients had a more favorable prognosis than patients with a normal BMI (7). In a study composed of 970 clear cell RCC patients, Parker et al. reported that high BMI was associated with negative lymph nodes and the absence of metastases (5).

Some hypotheses had been proposed to explain the contradiction. Patients with higher BMI might have better nutritional status and potential survival advantage (22). RCC developed in the obese might represent biologically distinct and less aggressive tumors versus those with normal weight $(6,23)$. Furthermore, patients with higher BMI were more likely to have contact with their physicians and have increased possibilities of early cancer detection (5).

The discovery of a new paradox inside the abovementioned paradox made the issue even more complicated. Bagheri et al. discovered through 8 studies of 8699 survivals that while CSS increased with BMI, when BMI is higher than 25, OS surprisingly decreased with BMI. Different causes of mortality had different directions after BMI reached a certain level, creating a 'paradox within a paradox' (24).

If we are to truly understand the role that BMI plays in RCC and other cancer patient, efforts are still needed to explicitly illustrate the issue in the future.

\section{Systemic inflammation state and RCC prognosis}

NLR has been recognized as the representative hematological index of systemic inflammation (14). However, studies about the prognostic value of the pretreatment NLR in non-metastatic $\mathrm{RCC}$ are sparse and with conflicting findings. Ohno et al. found that an increased NLR was an independent risk predictor for relapse-free survival in a small cohort of 192 RCC patients from Japan (25). Interestingly, Pichler et al. demonstrated that an increased NLR was an independent negative predictor for OS (26). Variance in study designs and sample sizes might bring about different outcomes. Considering the uncertainty of NLR's role in RCC patients, $\mathrm{Hu}$ performed a meta-analysis to assess the prognostic significance of high NLR for OS and RFS/PFS (progress-free survival), and found elevated NLR predicted poorer OS and RFS/ PFS in patients with RCC (27). 
As an index of systemic inflammation, high NLR might represent an inflammatory microenvironment which can increase mutation rates, in addition to enhancing the proliferation of mutated cells (28). High NLR is associated with high infiltration of tumor-associated macrophages (TAMs) which are identified to mediate refractoriness to anti-vascular endothelial growth factor (VEGF) treatment (29). Thus, that elevated NLR is related to poorer prognostic outcome of patients with RCC sounds reasonable. In Hu's meta-analysis, pooled analysis of studies showed NLR played a far more superior prognostic role with a cutoff value no more than 3 compared to higher than 3 (27). So, choosing 2.12 as the cutoff value seems applicable (In our study, the optimal cutoff value of NLR was 2.12 for CSS, calculated by ROC curve).

In this research, NLR was associated with OS and CSS in total patients in univariate and multivariate analyses, which showed that high NLR (systemic inflammation state) was independently associated with poor OS and CSS. Therefore, our findings are in support of the recognition that systemic inflammation state has a correlation with poor outcomes in RCC patients.

One aspect of importance is to distinguish systemic inflammation from chronic inflammation common in all obese people. Chronic inflammation happens because adipose tissue secretes pro-inflammatory cytokines, leading to a state of chronic low-grade inflammation associated with obesity, such that obese persons often experience higher concentrations of inflammatory biomarkers than their normal-weight counterparts (30). Systemic inflammation state in our research, however, is defined by high NLR $(>2.12)$, which is a much more severe condition than chronic inflammation above.

Prognostic value of BMI in a systemic inflammation state

As can be inferred from discussion above, the prognostic value of BMI was greatly enhanced in a systemic inflammation state. Systemic inflammation state makes possible a favorable environment for cancer cells: infiltration and metastasis are relatively easier (31). Therefore, cancer patients will become particularly sensitive in this condition and react more fiercely to changes in a lot of conditions. BMI is one example. As discussed above, patients with higher BMI tend to be in better nutritional conditions and vice versa; the influence of BMI on prognosis is exponentially magnified in a systemic inflammation condition. The results of our study validated this hypothesis (Figure-5). In subgroup analyses of our study, BMI was an independent favorable factor for CSS and RFS in high NLR (systemic inflammation state) patients rather than in low NLR patients, which indicated that the prognostic value of BMI was increased in systemic inflammatory status.

Another possible explanation includes the change in the effects of proinflammatory cytokines in subjects with high BMI. This is the case because obese patients are known to be associated with a state of chronic inflammation (23). Cytokines including C-reactive protein (CRP), tumor necrosis factor (TNF), IL6 and IL18, among others already increased greatly these patients (32). When this is the case, the effect of systemic inflammation (marked by high NLR) is attenuated since the body is already accustomed to abundance in inflammation cytokines. Therefore, the difference between survival outcomes between high and low BMI patients is further magnified, making it an especially sensitive independent predictor in a high NLR environment.

There are still some limitations in our study. As a retrospective study, selection bias was inevitable for making certain inclusion criteria. Another limitation was the relatively small size of samples from one single medical center. Our findings should be interpreted with caution until they are validated in a large multi-institutional pooled analysis.

If our finding that higher NLR increases the prognostic value of BMI is confirmed, BMI and NLR might need to be incorporated into the equation when protocols for therapy in RCC patients are planned.

\section{CONCLUSIONS}

In localized RCC patients, BMI was an independent favorable factor for CSS. In subgroup 
analyses, BMI was an independent protective factor for CSS in high NLR patients rather than in low NLR patients, which indicated that the prognostic value of BMI was increased in systemic inflammatory status.

\section{ACKNOWLEDGEMENTS}

We would like to thank all of the treating physicians of Peking University First Hospital for allowing us to include their patients.

Zhenhua Liu and Haifeng Wang contributed equally to this research and should be both considered first author.

\section{CONFLICT OF INTEREST}

None declared.

\section{REFERENCES}

1. Siegel R, Ward E, Brawley 0, Jemal A. Cancer statistics, 2011: the impact of eliminating socioeconomic and racial disparities on premature cancer deaths. CA Cancer J Clin. 2011;61:212-36.

2. Zhang C, Li X, Hao H, Yu W, He Z, Zhou L. The correlation between size of renal cell carcinoma and its histopathological characteristics: a single center study of 1867 renal cell carcinoma cases. BJU Int. 2012;110(11 Pt B):E481-5.

3. Calle EE, Rodriguez C, Walker-Thurmond K, Thun MJ. Overweight, obesity, and mortality from cancer in a prospectively studied cohort of U.S. adults. N Engl J Med. 2003:348:1625-38.

4. Samanic C, Chow WH, Gridley G, Jarvholm B, Fraumeni JF Jr. Relation of body mass index to cancer risk in 362,552 Swedish men. Cancer Causes Control. 2006;17:901-9.

5. Parker AS, Lohse CM, Cheville JC, Thiel DD, Leibovich BC, Blute ML. Greater body mass index is associated with better pathologic features and improved outcome among patients treated surgically for clear cell renal cell carcinoma. Urology. 2006;68:741-6.

6. Haferkamp A, Pritsch M, Bedke J, Wagener N, Pfitzenmaier $J$, Buse $S$, et al. The influence of body mass index on the long-term survival of patients with renal cell carcinoma after tumour nephrectomy. BJU Int. 2008;101:1243-6.
7. Kamat AM, Shock RP, Naya Y, Rosser CJ, Slaton JW, Pisters LL. Prognostic value of body mass index in patients undergoing nephrectomy for localized renal tumors. Urology. 2004;63:46-50.

8. Schips L, Lipsky K, Zigeuner R, Gidaro S, Salfellner M, Rehak $\mathrm{P}$, et al. Does overweight impact on the prognosis of patients with renal cell carcinoma? A single center experience of 683 patients. J Surg Oncol. 2004;88:57-61.

9. Donat SM, Salzhauer EW, Mitra N, Yanke BV, Snyder ME, Russo P. Impact of body mass index on survival of patients with surgically treated renal cell carcinoma. J Urol. 2006;175:46-52.

10. Lou M, Luo P, Tang R, Peng Y, Yu S, Huang W, et al. Relationship between neutrophil-lymphocyte ratio and insulin resistance in newly diagnosed type 2 diabetes mellitus patients. BMC Endocr Disord. 2015;15:9.

11. Keizman D, Ish-Shalom M, Huang P, Eisenberger MA, Pili $\mathrm{R}$, Hammers $\mathrm{H}$, et al. The association of pre-treatment neutrophil to lymphocyte ratio with response rate, progression free survival and overall survival of patients treated with sunitinib for metastatic renal cell carcinoma Eur J Cancer. 2012;48:202-8.

12. Donskov F, von der Maase H. Impact of immune parameters on long-term survival in metastatic renal cell carcinoma. J Clin Oncol. 2006;24:1997-2005.

13. Atzpodien J, Royston P, Wandert T, Reitz M; DGCIN -- German Cooperative Renal Carcinoma ChemoImmunotherapy Trials Group. Metastatic renal carcinoma comprehensive prognostic system. $\mathrm{Br} \mathrm{J}$ Cancer. 2003;88:348-53.

14. Roxburgh CS, McMillan DC. Role of systemic inflammatory response in predicting survival in patients with primary operable cancer. Future Oncol. 2010;6:149-63.

15. Fox P, Hudson M, Brown C, Lord S, Gebski V, De Souza P, et al. Markers of systemic inflammation predict survival in patients with advanced renal cell cancer. $\mathrm{Br} \mathrm{J}$ Cancer. 2013;109:147-53.

16. Choi Y, Park B, Jeong BC, Seo SI, Jeon SS, Choi HY, et al. Body mass index and survival in patients with renal cell carcinoma: a clinical-based cohort and meta-analysis. Int J Cancer. 2013;132:625-34.

17. Reynolds K, Gu D, Whelton PK, Wu X, Duan X, Mo J, et al. Prevalence and risk factors of overweight and obesity in China. Obesity (Silver Spring). 2007;15:10-8.

18. Chow WH, Gridley G, Fraumeni JF Jr, Järvholm B. Obesity, hypertension, and the risk of kidney cancer in men. N Engl J Med. 2000;343:1305-11. 
19. Jeon HG, Jeong IG, Lee JH, Lee CJ, Kwak C, Kim HH, et al. Prognostic value of body mass index in Korean patients with renal cell carcinoma. J Urol. 2010;183:448-54.

20. Awakura $\mathrm{Y}$, Nakamura $\mathrm{E}$, Ito $\mathrm{N}$, Yamasaki $\mathrm{T}$, Kamba $\mathrm{T}$, Kamoto $T$, et al. Influence of body mass index on prognosis of Japanese patients with renal cell carcinoma. Urology. 2007;70:50-4

21. Yu ML, Asal NR, Geyer JR. Later recurrence and longer survival among obese patients with renal cell carcinoma. Cancer. 1991;68:1648-55.

22. Choi Y, Park B, Jeong BC, Seo SI, Jeon SS, Choi HY, et al. Body mass index and survival in patients with renal cell carcinoma: a clinical-based cohort and meta-analysis. Int $\mathrm{J}$ Cancer. 2013;132:625-34.

23. Donin NM, Pantuck A, Klöpfer P, Bevan P, Fall B, Said J, et al. Body Mass Index and Survival in a Prospective Randomized Trial of Localized High-Risk Renal Cell Carcinoma. Cancer Epidemiol Biomarkers Prev. 2016;25:1326-32.

24. Bagheri M, Speakman JR, Shemirani F, Djafarian K. Renal cell carcinoma survival and body mass index: a dose-response meta-analysis reveals another potential paradox within a paradox. Int J Obes (Lond). 2016;40:1817-22.

25. Ohno $\mathrm{Y}$, Nakashima J, Ohori M, Hatano T, Tachibana M. Pretreatment neutrophil-to-lymphocyte ratio as an independent predictor of recurrence in patients with nonmetastatic renal cell carcinoma. J Urol. 2010;184:873-8.

26. Pichler M, Hutterer GC, Stoeckigt C, Chromecki TF, Stojakovic T, Golbeck S, et al. Validation of the pre-treatment neutrophil-lymphocyte ratio as a prognostic factor in a large European cohort of renal cell carcinoma patients. $\mathrm{Br} \mathrm{J}$ Cancer. 2013;108:901-7.
27. Hu K, Lou L, Ye J, Zhang S. Prognostic role of the neutrophillymphocyte ratio in renal cell carcinoma: a meta-analysis. BMJ Open. 2015;5:e006404

28. Grivennikov SI, Greten FR, Karin M. Immunity, inflammation, and cancer. Cell. 2010;140:883-99.

29. Mano $Y$, Shirabe K, Yamashita $Y$, Harimoto N, Tsujita E, Takeishi K, Aishima S, et al. Preoperative neutrophil-tolymphocyte ratio is a predictor of survival after hepatectomy for hepatocellular carcinoma: a retrospective analysis. Ann Surg. 2013;258:301-5.

30. Ferrante AW Jr. Obesity-induced inflammation: a metabolic dialogue in the language of inflammation. J Intern Med. 2007;262:408-14.

31. Hu H, Yao X, Xie X, Wu X, Zheng C, Xia W, et al. Prognostic value of preoperative NLR, dNLR, PLR and CRP in surgical renal cell carcinoma patients. World J Urol. 2017;35:261-70.

32. Osborn 0, Olefsky JM. The cellular and signaling networks linking the immune system and metabolism in disease. Nat Med. 2012;18:363-74.

Correspondence address: Jie Jin, MD, PhD

Department of Urology, Peking University First Hospital and Institute of Urology, Peking University, 8 Xishiku Street, Xicheng District, Beijing 100034, China. E-mail: jinjie@vip.163.com 


\section{APPENDIX - Supplementary Figures}

Figure-S.1 - Receiver operating characteristic (ROC) curve for BMI.

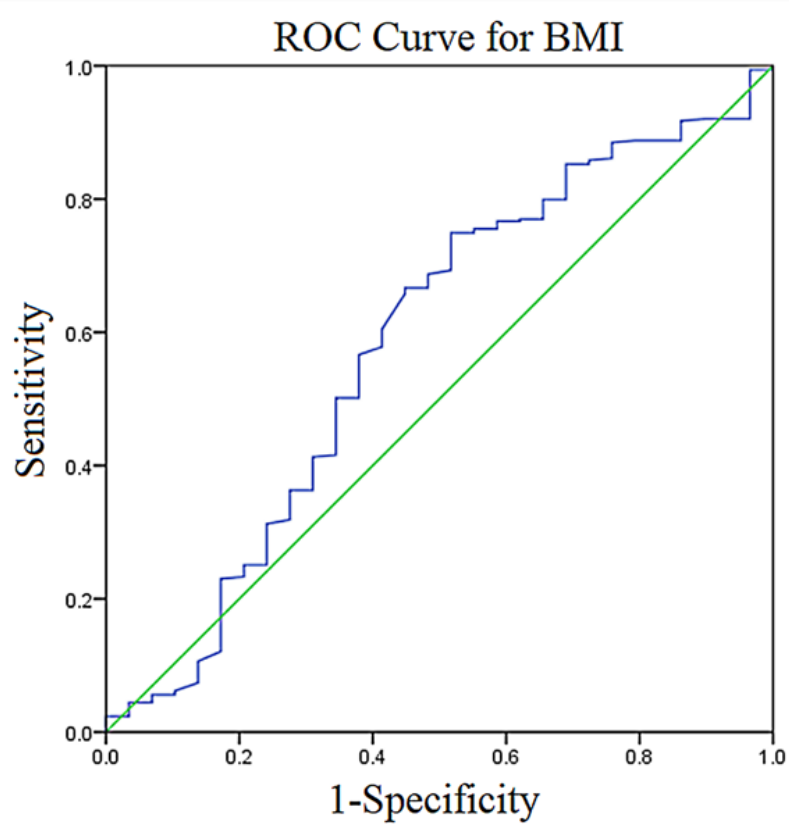

Abbreviations: $\mathbf{B M I}=$ body mass index.
Figure-S.2 - Receiver operating characteristic (ROC) curve for NLR.

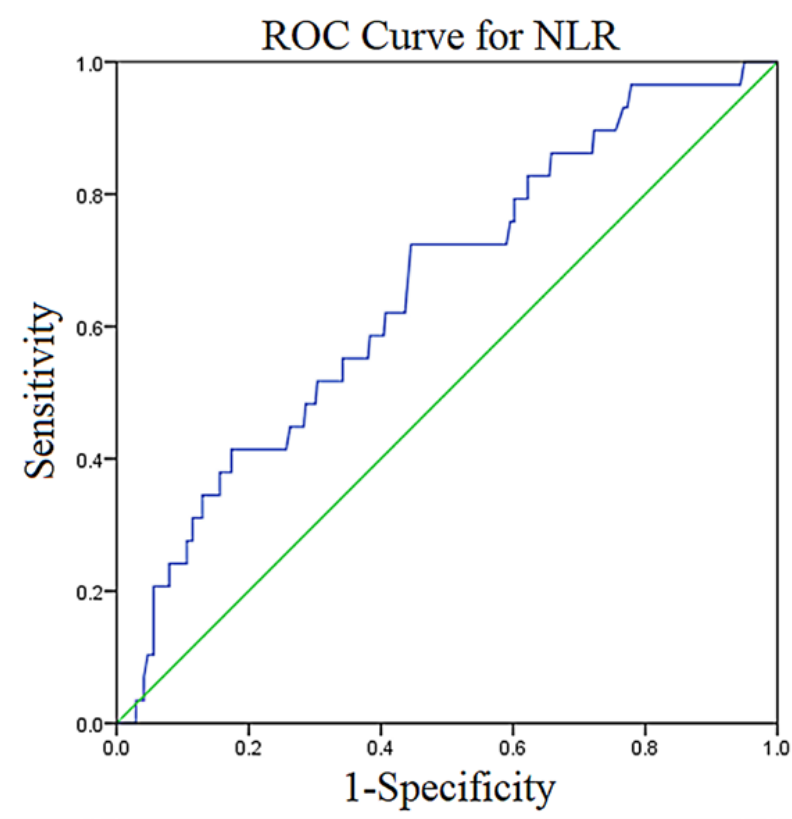

Abbreviations: NLR=neutrophil-lymphocyte ratio.

Figure-S.3 - Survival curves stratified by BMI at the level of 23.32 in low NLR patients (Kaplan-Meier method).

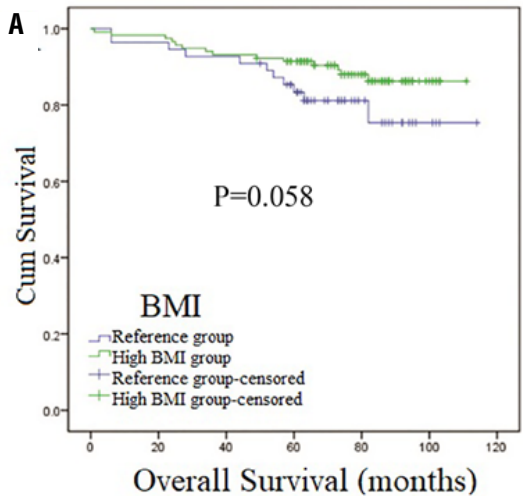

Overall Survival (months)

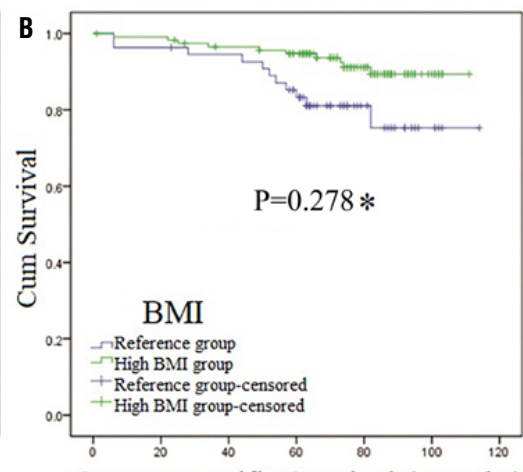

Cancer-specific Survival (months)

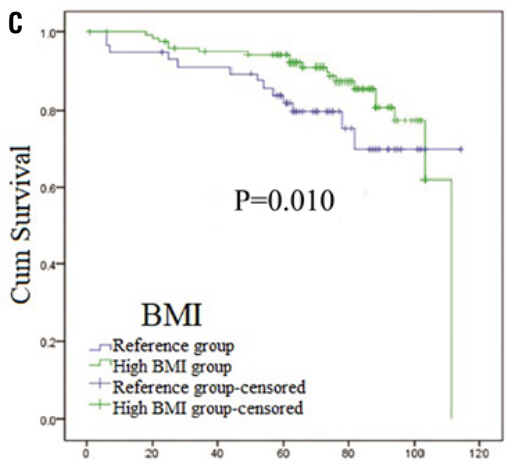

Recurrence-free Survival (months)

Survival curves of OS (Figure S.3A), CSS (Figure S.3B) and RFS (Figure S.3C) in low NLR patients.

Abbreviations: $\mathbf{B M I}=$ body mass index; $\mathbf{N L R}=$ neutrophil-lymphocyte ratio.

$*: p<0.0$ 